\title{
The role of scanpaths in the recognition of random shapes*
}

\author{
PAUL J. LOCHER $†$ and CALVIN F. NODINE \\ Temple University, Philadelphia, Pennsylvania 19104
}

\begin{abstract}
Eye movements of 12 Ss were examined during learning and recognition of two-dimensional random shapes to determine the nature of the memorial representation of a stimulus and the utilization of this memorial reprecentation in pattern recognition. Specifically, the purpose of this study was to test the scanpath model of pattern perception by determining whether scanpaths exist and, if so, how they influence recognition performance. Scanpaths, defined as overlapping fixation patterns in learning and recognition tasks, were observed in over half of all eye-movement records regardless of shape complexity. Presence of scanpaths did not increase recognition performance as measured by errors in recognition and $\mathbf{S}$ ' ability to reproduce the shapes. Although scanpaths did not influence recognition performance, their occurrence implicates them as a potential factor in the recognition process.
\end{abstract}

Noton (1970) has proposed a theory of visual pattern perception in which the processing of pattern features is viewed as a serial process with a fixed-order strategy of extraction of information from the visual display during both memorization and recognition. The theory is based upon the assumption that learning to recognize a pattern consists first of constructing an internal representation of that pattern, storing it in memory, and second, recognizing this pattern by matching it to its memorial representation (Noton, 1970). Several theorists have emphasized the sequential nature of pattern perception (e.g., Hebb, 1949; Hochberg, 1968). However, as Noton points out, disagreement concerning the nature of the perceptual process centers around the specific nature of the memorial representation of the stimulus and the utilization of this memorial representation in pattern recognition.

With regard to the strategy used to extract and encode information from the stimulus display, Noton has proposed that each stimulus is represented in memory as a sequence of sensory memory traces which form a "feature ring" containing both the structural elements of the stimulus pattern and the attention shifts required to move from one structural feature to the next. During recognition, the feature elements of a stimulus pattern are sequentially matched against memory traces in feature networks until a match is achieved on a point-by-point basis in terms of attention shifts called scanpaths between the stimulus pattern and appropriate feature network.

Studies which have examined eye movements as a form of observable attention shifts (Gould, 1967; Gould \& Dill, 1969; Yarbus, 1967) have demonstrated the presence of scanpaths or cyclical repetitions of fixations in the scanning patterns of Ss memorizing visual stimuli. However, as Noton (1970) points out, the use of these scanpaths in subsequent recognition awaits

*The present paper is adapted from a dissertation submitted to Temple University by the first author.

+Now at Montclair State College, Department of Psychology, Upper Montclair, New Jersey 07043. Requests for reprints should be sent to the first author. confirmation. The purpose of this study was to test the scanpath model of pattern recognition by determining whether scanpaths exist and, if so, how they influence recognition performance.

\section{METHOD}

\section{Subjects}

Twelve undergraduate college students (six males and six females) enrolled in an introductory psychology class at Temple University served as $\mathbf{S s}$. Each $\mathbf{S}$ was paid for his participation.

The eye-movement record for each of the $12 \mathrm{Ss}$ was complete, and the definition and contrast of each of the filmed records was of sufficient quality so that none of the data was lost.

\section{Stimulus Materials}

Eighteen of the 38 straight-line two-dimensional geometric shapes used by Vitz and Tadd (1971) were used as stimuli. The shapes, with their identification numbers and with their total figure complexity values as calculated by Vitz and Todd, are shown in Fig. 1.

These particular shapes were selected on the general intuitive grounds that the set of 18 shapes represented a wide variety of structural characteristics and a wide range of total figure complexities.

The shapes for this study were prepared directly from Figs. 4 and 6 in Vitz and Todd (1971, pp. 215 and 218). The orientation and area of each shape relative to the set of shapes were carefully maintained. The shapes were made of $3 / 32$-in. black matte tape mounted on $14 \times 12$ in. sheets of clear plastic and then reproduced on $35-\mathrm{mm}$ slides.

\section{Apparatus}

The shapes were presented to $S$ via a frosted rear-projection screen using a Kodak Carousel projector positioned $121.92 \mathrm{~cm}$ from the screen. The display on the screen consisted of a single, white, straight-lined, two-dimensional shape on a black background. The rear-projection screen was attached to a Polymetric eye-movement recorder, wide-angle (Model V-1166). This apparatus was equipped with a 16-mm Beaulieu motion-picture camera with Soligon telephoto lens and Paillard-Bolex 40-mm extension tube.

The stimulus display was reflected off the right eye of $S$ as he examined the display. The eye, with superimposed picture of the display, was photographed at $8 \mathrm{fps}$. Figure 2 presents one frame of a filmed record.

Attached to the eye-movement apparatus was a chin- and headrest to help hold the S's head in position as he examined the 


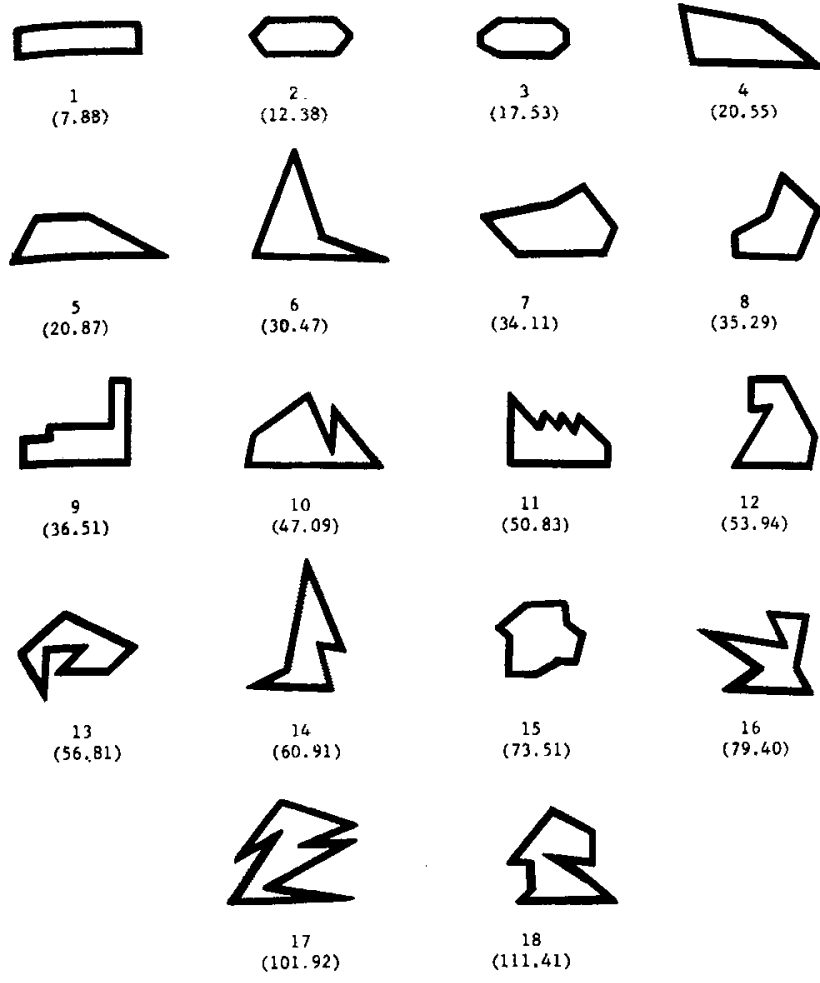

Fig. 1. Stimulus shapes. The number below each shape is its identification number, and the number in parenthesis is that shape's total figure complexity value as calculated by Vitz and Todd (1971).

stimulus display. The recording accuracy of the wide-angle eye camera has been shown to fall within \pm 2 deg of visual angle (Kundel \& Nodine, 1973), to detect multiple fixations on each shape. A shape projected on the screen subtended approximately $36 \mathrm{deg}$ horizontally $(22.86 \mathrm{~cm})$ and approximately $24 \mathrm{deg}$ vertically $(15.24 \mathrm{~cm})$. By dividing the display area into 4-deg grid squares (based upon the recording accuracy of $\pm 2 \mathrm{deg}$ ), it was possible to obtain as many as 54 individual fixations per shape.

\section{Data Reduction}

The position of each S's fixations was determined by projecting the filmed records, one frame at a time, onto the surface of a Thomson table on line with a LINC-8 computer and locating the $x$ and $y$ coordinates of the stimulus shape relative to the fovea. This was accomplished by first placing a cursor over the center of the display, feeding the $x-y$ coordinates of this , point (in digital form) into the computer, and then placing the cursor over the center of the pupil and feeding the $x-y$ coordinates of this point into the computer. The computer was programmed to normalize these coordinates, which were then stored on maghetic tape.

Each frame of the filmed record was examined in sequence in order to determine the locus of gaze. For each $\mathrm{S}$ viewing each shape, the computer was programmed to output the number and sequence of fixations, the $x-y$ coordinates of each fixation, and the fixation time (in frames of film) per fixation.

\section{Experimental Procedure}

The experimental procedure consisted of three phases, which took each $\mathrm{S}$ approximately $30 \mathrm{~min}$ to complete. The first phase was a "learning phase," and Phases 2 and 3 were "Recognition Phase 1" and "Recognition Phase 2," respectively. Filmed records were made for each $S$ during each of the three phases.

Learning Phase. During the learning phase, each S was directed to focus his attention upon a dot that was displayed in the center of the projection screen. The dot subtended 1 deg. This dot was then replaced by a polygon, and $S$ was instructed to examine the polygon for as long as was necessary to "remember" the shape. The $S$ was directed to close his eyes as soon as he had memorized the shape. Immediately after viewing a shape, $\mathbf{S}$ was asked to draw the shape on a blank $8 \times 11$ in. piece of paper. The importance of the accu racy of each drawing was emphasized to the Ss, and no indication of the amount of time that should be spent on a particular shape was included in the instructions to Ss.

During the learning phase, each $S$ examined one of three random orders of the following 12 shapes (see Fig. 1): Nos. 1, 3, $4,6,7,9,10,12,13,15,16$, and 17 .

Recognition Phase 1. Twelve shapes comprised the first recognition phase. For each S, 6 of these were "old" shapes randomly selected from the 12 learning phase stimuli, and the remaining 6 shapes, Nos. 2, 5, 8, 11, 14, and 18, were "new" ones which $S$ had not seen before. These 12 shapes (half old, half new) comprised the first recognition phase for that $S$. Each of the 12 learning phase stimuli appeared an equal number of times in Recognition Phase 1 across all Ss; however, only 6 learning phase stimuli were included in any one S's set of Recognition Phase 1 stimuli.

The shapes in Recognition Phase 1 were presented in random order one after the other, allowing $S$ only enough time (about $2 \sec )$ to respond "yes" if he had seen that shape during the learning phase or "no" if the shape was new to him. As in the learning task, the importance of the correctness of response was emphasized and Ss were given no indication of the amount of time which should be spent examining a particular shape. Each shape was preceded by a dot located in the center of the screen.

Recognition Phase 2. Each S's Recognition Phase 1 set of shapes was randomly rearranged to make up that S's set of shapes for the second recognition phase. The Recognition Phase 2 shapes were presented to $S$ in exactly the same manner as in the earlier recognition phase. Again, $S$ was asked to respond "yes" if he had seen the shape before, during the learning phase, or "no" if he had not seen the shape during the learning phase.

\section{RESULTS}

\section{Analysis of Scanpaths}

The data were examined to determine if repetitive

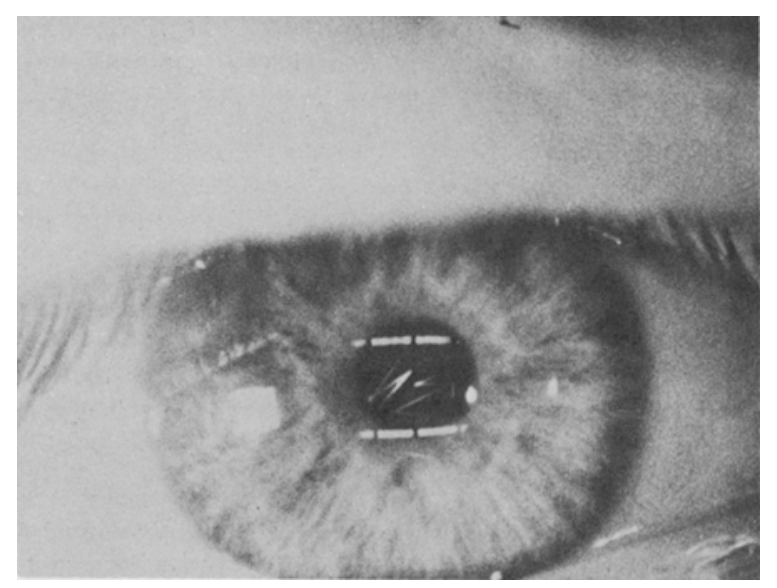

Fig. 2. One frame of a filmed record showing the stimulus display (Shape 17) reflected on the cornea of an $S$ viewing the display. 


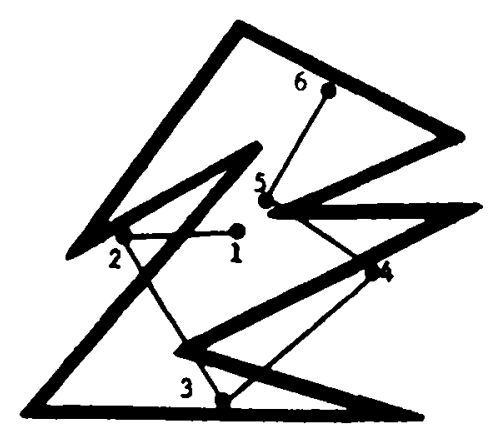

$\mathbf{A}$

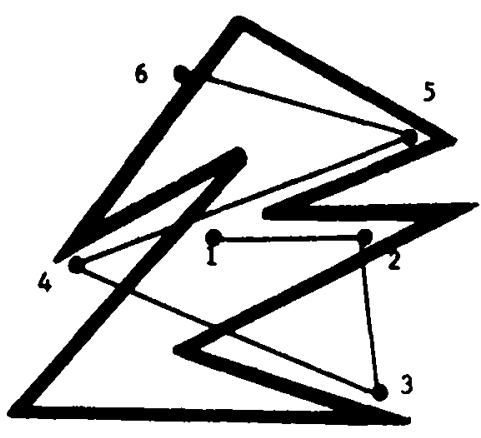

D

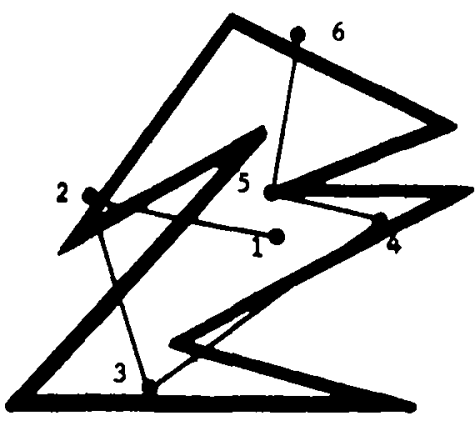

B

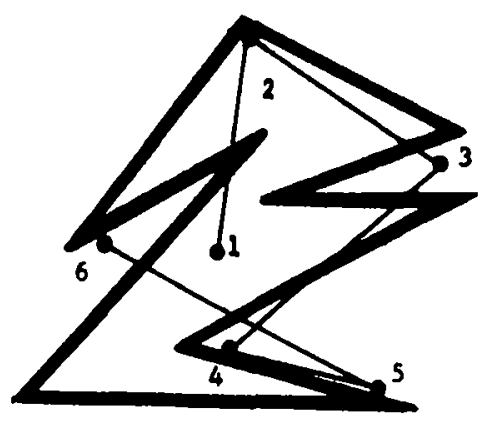

E

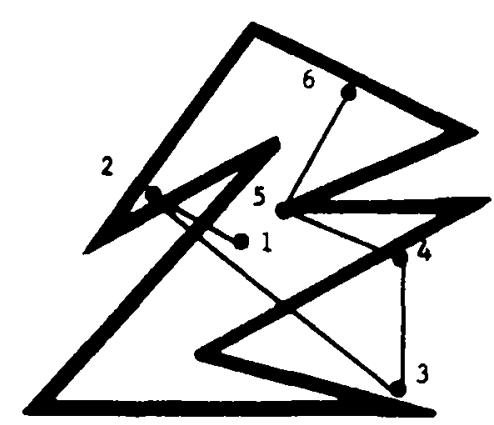

c

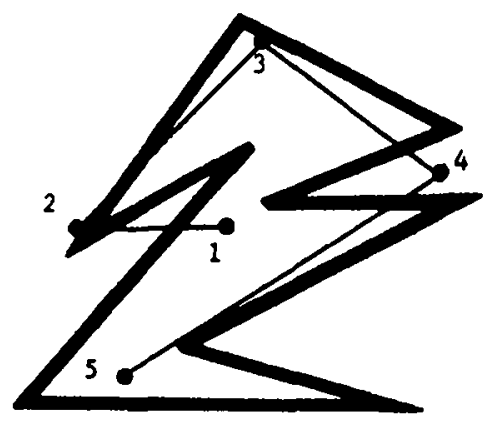

$\mathbf{F}$

Fig. 3. Examples of eye-movement patterns illustrating the presence or absence of a scanpath. Top row shows scanning pattern for $S_{1}$ for Shepe 17 in learning (A), Recognition 1 (B), and Recognition 2 (C). Bottom row shows scanning pattern for $S_{2}$ for Shape 17 for learning (D), Recognition 1 (E), and Recognition 2 (F). Scanpath is present in $S_{1}$ (top row) and absent in $S_{2}$ (bottom row). Fixations for each scanning pattern are numbered sequentially.

paths or sequences of fixations (scanpaths) could be observed in Ss' fixation patterns between learning and both recognition phases and between Recognition Phase 1 and Recognition Phase 2. In order to test the scanpath model, the percentage of fixation patterns exhibiting scanpaths was contrasted with the chance occurrence of these sequences of fixations and with the findings of the only other studies (Noton \& Stark, $1971 \mathrm{a}, \mathrm{b})$ which have systematically attempted to document the presence of scanpaths in eye-movement records.

Scanpaths Between Learning and Recognition. For purposes of the present analysis, scanpaths were defined in the following way. The position and sequence of all fixations for a given $S$ and given shape during Recognition Phase 1 were plotted. As an exmple, six fixations were recorded for $S_{1}$ for Shape 17 during Recognition Phase 1. These six fixations were compared with the first six fixations for $S_{1}$ for Shape 17, recorded during the learning phase. Figure 3 presents the fixation patterns for $S_{1}$ (scanpath present) and $S_{2}$ (no scanpath present) for Shape 17. In making such comparisons, the first fixation from Recognition Phase 1 was compared with the first fixation from the learning phase. If both fixations fell within 2 deg of visual angle of each other, it was concluded that the same area of the stimulus display was initially examined during the performance of both tasks. Each of the remaining fixations of Recognition Phase 1 was compared to its corresponding learning phase fixation. The second recognition phase fixation was compared with the second learning phase fixation, and so on through the sequence of Recognition Phase 1 fixations.

A scanning pattern for a particular $S$ examining a particular shape was said to exhibit a scanpath if $50 \%$ of the fixations for a given shape from the recognition phase fell within the same areas of the stimulus display as the correspondingly numbered fixations recorded during the learning phase.

The same procedure was used to detect the presence of scanpaths between learning and Recognition Phase 2 . In this case, the first Recognition Phase 2 fixation was compared to the first learning phase fixation, and if both fixations fell within 2 deg of visual angle of each other, it was concluded that the same area of the stimulus display was initially examined during the performance of both tasks.

Scanpaths Between Recognition Phases. The scanning patterns of the shapes (new) seen for the second time during Recognition Phase 2 were also examined for the 
Table 1

Mean Number of Scanpaths Per S and Per Shape Occurring During Recognition Phases 1 and 2

\begin{tabular}{|c|c|c|c|c|c|c|}
\hline & \multicolumn{2}{|c|}{$\begin{array}{l}\text { Recognition } \\
\text { Phase } 1 \\
\text { (Old Shapes) }\end{array}$} & \multicolumn{2}{|c|}{$\begin{array}{l}\text { Recognition } \\
\text { Phase } 2 \\
\text { (Old Shapes) }\end{array}$} & \multicolumn{2}{|c|}{$\begin{array}{l}\text { Recognition } \\
\text { Phase } 2 \\
\text { (New Shapes) }\end{array}$} \\
\hline & Mean & SD & Mean & SD & Mean & SD \\
\hline $\begin{array}{l}\text { Mean number of scanpaths per } S \text { for six shapes } \\
\text { Number of Ss showing more }(+) \text { and fewer }(-) \text { scanpaths than mean }\end{array}$ & $\begin{array}{l}2.75 \\
7(+) \\
5(-)\end{array}$ & 1.54 & $\begin{array}{l}3.50 \\
8(+) \\
4(-)\end{array}$ & 1.44 & $\begin{array}{l}4.25 \\
6(+) \\
6(-)\end{array}$ & 1.35 \\
\hline $\begin{array}{l}\text { Mean number of scanpaths per shape } \\
\text { Number of shapes showing more }(+) \text { and fewer }(-) \text { scanpaths than mean }\end{array}$ & $\begin{array}{l}2.91 \\
3(+)^{*} \\
9(-)\end{array}$ & .99 & $\begin{array}{l}3.35 \\
7(+) * * \\
5(-)\end{array}$ & 1.13 & $\begin{array}{l}8.50 \\
3(+) \dagger \\
3(-)\end{array}$ & 1.51 \\
\hline
\end{tabular}

*Six Ss contributed data for each shape. Shapes 3, 7, and 17 were above 3.

**Six Ss contributed data for each shape. Shapes 1, 3, 4, 12, 13, 16, and 17 were above 3.

tAll 12 Ss contributed data for six new shapes. Shapes 11, 14, and 18 were above 8.

presence of scanpaths. In this case, a S's fixation patterns for the new shapes seen for the first time during Recognition Phase 1 were compared with his fixation patterns for the new shapes seen for the second time in Recognition Phase 2. That is, the first Recognition Phase 2 fixation for a given new shape was compared to the first Recognition Phase 1 fixation for that shape. Scanpaths were defined in the same way as described previously.

It was found that $47 \%$ of all Recognition Phase 1 scanning patterns for old shapes exhibited scanpaths; $57 \%$ of all Recognition Phase 2 scanning patterns for old shapes exhibited scanpaths; and $71 \%$ of all Recognition Phase 2 scanning patterns for new shapes exhibited scanpaths. The distributions of scanpaths across Ss and across shapes are presented in Table 1. Noton and Stark (1971b) found, using line drawings as stimuli, that $65 \%$ of the recognition task scanning patterns of Ss exhibited scanpaths. Noton and Stark, however, fail to provide an operational definition of scanpath.

Scanpaths have been observed in the data for both recognition phases. The question remains as to whether S's performance on a task was in any way related to the occurrence of scanpaths in his scanning patterns. Two measures of performance were examined, errors and drawings.

First, the scanning patterns of shapes mistakenly identified during both recognition phases were examined. A total of 20 mistakes (10\% of the responses) was made by 11 of the 12 Ss over 9 different shapes. In 15 cases, "old" shapes were mistakenly identified as "new" shapes. The scanning patterns for 8 of these shapes exhibited scanpaths; 7 did not. The remaining five mistakes in identification were for "new" shapes identified as "old" shapes. Three of the scanning patterns for these shapes exhibited scanpaths; two did not. This finding suggests that the occurrence of a scanpath in the scanning pattern of a $S$ did not insure correct identification of a shape during a recognition task by that $S$, since the scanning patterns for 11 of the shapes mistakenly identified exhibited scanpaths, while the scanning patterns for the other 9 misidentified shapes did not exhibit scanpaths.

Second, the drawings that Ss completed during the learning phase were examined to see if the accuracy of a drawing (the amount of information reproduced) was related to the occurrence of a scanpath in the scanning pattern for the shape drawn.

Of the 12 learning phase shapes examined and consequently drawn by each S, 6 of the shapes were randomly selected for inclusion in the S's Recognition Phase 1 and 2 sets of stimuli. The drawings of these 6 (old) shapes for each $\mathrm{S}$ were examined for the accuracy of reproduction in the following way. The drawings of old shapes for all Ss were arranged in 12 piles, 1 pile for each of the 12 learning phase stimuli. Each pile contained six drawings. It should be remembered that each of the 12 learning phase stimuli appeared in only six Recognition Phase 1 and 2 sets of stimuli. Three raters were each asked to look at the six drawings of a particular shape and to rank the drawings on a 6-point scale as to the accuracy of the representation of the original shape ( 1 being the rank for the drawing which was the best reproduction of the original stimulus shape). Each rater ranked the six drawings in each pile. Interrater reliability on this task was .85 . Table 2 shows the distributions of the accuracy ratings for the drawings of all old shapes and the occurrence of scanpaths in the scanning patterns for these shapes.

For Recognition Phase 1, it was found that there was no relationship $\left(\chi^{2}=1.11\right.$, $\mathrm{df}=5$, n.s.) between the accuracy of S's drawing and the occurrence of a scanpath in that S's scanning pattern for the shape drawn. Similar results were obtained for Recognition Phase 2 data $\left(\chi^{2}=2.49, \mathrm{df}=5\right.$, n.s. $)$.

\section{Role of Shape Complexity in Recognition Phase 1}

The relationship between shape complexity and learning was determined using two eye-movement measures, number of frames per shape and the number of fixations per shape. A randomized block factorial design with repeated measures (Kirk, 1968) was used with both dependent measures. This design had two treatment variables. Two levels of learning experience 
Table 2

Two 2 by 6 Contingency Tables Showing the Distribution of the Accuracy of Representation of Stimuli and the Occurrence of Scanpaths in Scanning Patterns for Recognition Phases 1 and 2

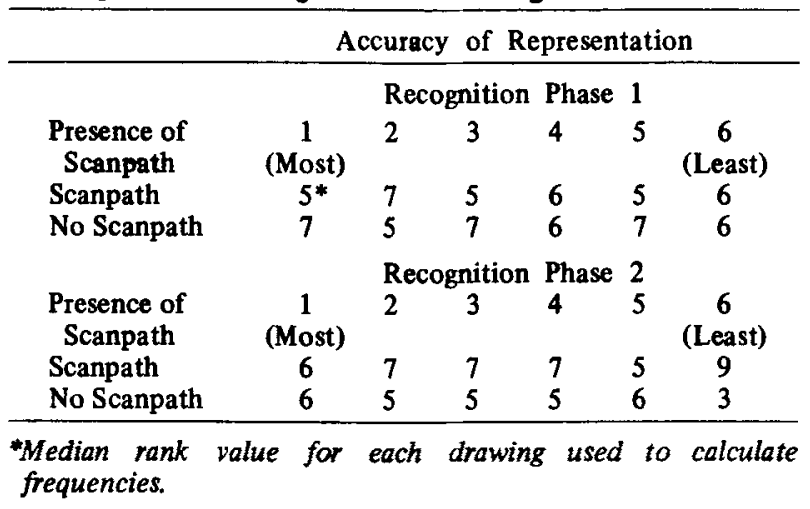

(E) with the shapes were "old" (learning experience) and "new" (no learning experience). Three levels of shape complexity (C) were low (scale values of 7.88-30.47), medium (scale values of 34.11-53.94), and high (scale values of 59.81-111.41). These are shown in Fig. 1.

$\log _{10}$ values of the mean number of frames for the two shapes at each complexity level for each $S$ served as the dependent measures for the first analysis of variance, and $\log _{10}$ values of the mean number of fixations for the two shapes at each complexity level for each $S$ served as the dependent measure for the second analysis of variance. $\log _{10}$ transformations of the data were necessary to achieve the symmetry of the variance-covariance matrices required for the use of analysis of variance with repeated measures. The means and standard deviations for the number of frames and the number of fixations for old and new shapes at each level of complexity are shown in Table 3.

With respect to viewing time (frames), no significant main effects were obtained for either learning experience (E) or shape complexity (C). However, the $\mathrm{E}$ by $\mathrm{C}$ interaction was significant $(F=5.26, \mathrm{df}=2,22, \mathrm{p}<.05)$.

In order to examine the nature of the interaction, the data were considered as two single-factor experiments of a randomized block design on Variable E (Winer, 1962). Using this technique, it was found that complexity had no effect on viewing time for new shapes in Recognition Phase 1. However, a significant effect of complexity was obtained for old shapes $(\mathrm{F}=5.14, \mathrm{df}=2,22, \mathrm{p}<.05)$. Viewing time increased with shape complexity for old shapes during Recognition Phase 1.
With respect to the number of fixations, again no significant main effects were obtained for either learning experience (E) or shape complexity (C). The E by $C$ interaction was significant $(\mathrm{F}=5.64, \mathrm{df}=2,22, \mathrm{p}<.05)$. An examination of this interaction indicated that, as with time, complexity had no effect on the number of fixations used to examine new shapes in Recognition Phase 1. However, there was a trend $(F=3.30$, $\mathrm{df}=$ $2,22, p=.06$ ) for fixations to increase directly with shape complexity for old shapes during Recognition Phase 1.

\section{Role of Shape Complexity in Recognition Phase 2}

An identical analysis was performed on both fixation time and number of fixation measures for Recognition Phase 2. The means and standard deviations for the number of frames and the number of fixations for old and new shapes at each level of complexity are shown in Table 4.

Only shape complexity (C) produced a significant effect $(F=8.26, d f=2,22, p<.01)$. A Tukey ratio test indicated that more time was used to examine shapes of both medium complexity $(\mathrm{q}=3.88, \mathrm{df}=3,22, \mathrm{p}<.05)$ and high complexity $(\mathrm{q}=5.76, \mathrm{df}=3,22, \mathrm{p}<.01)$ than shapes of low complexity, but there was no difference between the amount of time used to examine shapes of medium and high complexities.

The analysis of variance for the number of fixations yielded no significant main effect for either learning experience (E) or the $\mathrm{E}$ by $\mathrm{C}$ interaction. Level of complexity (C) again produced a significant effect $(F=$ $7.91, \mathrm{df}=2,22, \mathrm{p}<.01)$. It was found that during Recognition Task 2, Ss used more fixations examining shapes of medium $(q=3.75, \mathrm{df}=3,22, \mathrm{p}<.05)$ and high $(\mathrm{q}=5.54, \mathrm{df}=3,22, \mathrm{p}<.01)$ complexities than they did examining shapes of low complexity. There was no difference between medium and high complexities.

\section{DISCUSSION}

The findings of this study are equivocal with respect to the scanpath model of pattern recognition. If the perceptual process used in the recognition of patterns involves a sequential (serial) matching of features as directed by the scanpath model, the following temporal prediction can be made concerning the process. More complex patterns will take longer to recognize than simpler patterns, since more features must be examined.

Table 3

Recognition Phase 1 Means and Standard Deviations for the Number of Frames and Fixations in Each Treatment Level

\begin{tabular}{|c|c|c|c|c|c|c|c|c|c|c|c|c|}
\hline \multirow{4}{*}{$\begin{array}{c}\text { Learning } \\
\text { Experience }\end{array}$} & \multicolumn{12}{|c|}{ Complexity Levels } \\
\hline & \multicolumn{6}{|c|}{ Frames } & \multicolumn{6}{|c|}{ Fixations } \\
\hline & \multicolumn{2}{|c|}{ Low } & \multicolumn{2}{|c|}{ Medium } & \multicolumn{2}{|c|}{ High } & \multicolumn{2}{|c|}{ Low } & \multicolumn{2}{|c|}{ Medium } & \multicolumn{2}{|c|}{$\mathrm{High}$} \\
\hline & Mean & SD & Mean & SD & Mean & SD & Mean & $\mathrm{SD}$ & Mean & $\mathrm{SD}$ & Mean & SD \\
\hline Old Shapes & 13.16 & 6.71 & 18.04 & 9.84 & 21.04 & 13.00 & 7.00 & 3.63 & 9.00 & 6.74 & 11.33 & 6.73 \\
\hline New Shapes & 16.75 & 7.49 & 17.91 & 10.36 & 17.66 & 9.96 & 9.50 & 4.64 & 9.25 & 5.86 & 8.16 & 4.85 \\
\hline
\end{tabular}


Table 4

Recognition Phase 2 Means and Standard Deviations for the Number of Frames and Fixations in Each Treatment Level

Complexity Levels

\begin{tabular}{|c|c|c|c|c|c|c|c|c|c|c|c|c|}
\hline \multirow{4}{*}{$\begin{array}{l}\text { Learning } \\
\text { Experience }\end{array}$} & \multirow{2}{*}{\multicolumn{6}{|c|}{ Frames }} & \multirow{2}{*}{\multicolumn{6}{|c|}{ Fixations }} \\
\hline & & & & & & & & & & & & \\
\hline & \multicolumn{2}{|c|}{ Low } & \multicolumn{2}{|c|}{ Medium } & \multicolumn{2}{|c|}{ High } & \multicolumn{2}{|c|}{ Low } & \multicolumn{2}{|c|}{ Medium } & \multicolumn{2}{|c|}{ High } \\
\hline & Mean & SD & Mean & SD & Mean & SD & Mean & SD & Mean & SD & Mean & SD \\
\hline Old Shapes & 9.87 & 5.79 & 12.62 & 8.61 & 16.54 & 7.10 & 4.73 & 2.20 & 6.83 & 3.32 & 8.12 & 3.50 \\
\hline New Shapes & 11.25 & 4.57 & 15.45 & 7.07 & 19.25 & 12.88 & 5.83 & 3.33 & 8.95 & 4.40 & 8.61 & 4.41 \\
\hline
\end{tabular}

This was the case. It was found that there were no differences in viewing time or in the number of fixations used by Ss during Recognition Phase 1 for new shapes differing in structural complexity which they had not seen previously during the learning phase. However, these same Ss spent increasing amounts of viewing time and used increasing numbers of fixations examining old shapes during Recognition Phase 1. Moreover, both fixation time and the number of fixations increased directly with the complexity of the old shapes but not with new shapes.

According to the scanpth model, as $\mathrm{S}$ begins to extract information from the visual display during the recognition task, attention should be initially directed randomly to some structural feature of the shape. ${ }^{1}$ If one of these early fixations activates an existing memory trace constructed during the learning phase, the recognition system will execute a sequence of attentional shifts specified by the scanpath.

When examining old shapes during Recognition Phase 1, fixation time and the number of fixations increased directly with the complexity of the old shape. This finding is consistent with the prediction of serial models that reaction time in recognition tasks increases with the number of relevant dimensions of the stimulus (Egeth, 1966). Since total shape complexity is a measure of the relevant dimensions comprising a shape, the number of fixations and the amount of fixation time should increase directly with total shape complexity if the perceptual sampling process used in recognition is serial.

When examining new shapes during Recognition Phase 1, $\mathrm{S}$ began to examine the dimensions of a particular shape, and failing to find in memory a potential match for a learning pattern, terminated the search and responded after only a few fixations. Because Ss used the same number of fixations and equal amounts of time examining all new shapes regardless of complexity, it was concluded that the search process was independent of stimulus complexity and self-terminated after a brief testing of features.

During Recognition Phase 2, Ss spent an equal amount of time and used equal numbers of fixations examining all shapes of a given complexity level regardless of whether Ss had seen the shapes during the learning phase or not. During Recognition Phase 2, however, the Ss did spend more time and use more fixations examining all shapes of medium and high complexities than they did examining shapes of low complexity. These findings differed from those obtained for Recognition Phase 1 data. During Recognition Phase 1 , Ss used equal numbers of fixations and equal amounts of time examining new shapes regardless of the level of complexity of the shapes. The difference is most consistent with a serial/fixed-order/self-terminating strategy.

New shapes were, in fact, "new" to $S$ during Recognition Phase 1 because $S$ had not seen them during the learning phase. No internal representation (scanpath) existed for these shapes which could have been used in making comparisons during Recognition Phase 1. Therefore, the search process for all new shapes, regardless of complexity, was terminated quickly during Recognition Phase 1. However, during Recognition Phase 2, "new" shapes were "old" in the sense that they had been examined during Recognition Phase 1, since Recognition Phase 2 sets of stimuli were merely rearranged Recognition Phase 1 sets of stimuli. Accordingly, if a scanpath for a new shape was set down during Recognition Phase 1, then when S encountered that "new" shape (now really old) again during Recognition Phase 2, he used the same search process as used in Phase 1. Both number of fixations and the amount of fixation time should, according to a serial model of pattern recognition, increase with the number of relevant dimensions of the stimulus as expressed by the total figure complexity of a shape. This was the case for both old and "new" (now really old) shapes during Recognition Phase 2.

The scanpath model suggests an efficient and accurate utilization of stimulus information during recognition. This accuracy should manifest itself in the task performance of Ss for whom scanpaths were observed. It seemed reasonable to assume, therefore, that if a scanpath existed in the recognition phase scanning pattern for a particular shape, $S$ should have correctly identified that shape. However, this was not the case. Of the 20 shapes which were misidentified by all Ss during both recognition phases, one-half of the eye-movement records for these shapes exhibited scanpaths. Thus, presence of a scanpath in the scanning pattern for a shape did not influence the probability of a correct identification of that shape.

It also seemed reasonable to assume that the drawings 
of those shapes for which subsequent scanpaths were observed should have been better representations of the original stimuli than the drawings of shapes for which no scanpaths were observed in the scanning patterns. This was not borne out. For both Recognition Phase 1 and 2 data, there was no relationship between the judged accuracy of a S's drawing and the occurrence of a scanpath in that $S$ 's eye-movement record.

Concerning the relationship between the accuracy of task performance and the presence of scanpaths, the above findings may reflect possible limitations of the present study. One such limitation may be the attenuated range of structural complexity of the shapes used as stimuli. Evidence of this is indicated by the fact that only $10 \%$ errors occurred in recognition. Also, sufficient emphasis upon the accuracy of responses was not structured in the design of the task.

This study was designed to force Ss to scan the stimuli so as to make available for measurement part of the visual pattern recognition process. Noton (1970) has pointed out that it might be possible that eye movements are simply controlled by peripheral visual input and by higher level control, such as a feature network. Little is known about the role of peripheral vision in pattern perception. This is clearly an area in which much research is needed. While peripheral vision is unquestionably an important factor in the activation of sensory memory traces, it seems unlikely that peripheral vision alone can provide sufficient resolution to identify complex stimuli without the aid of foveal vision checks (Nodine \& Steuerle, 1973).

With respect to the normal viewing of small pictures and stimuli presented tachistoscopically, when recognition requires few or no eye movements, the scanpath model assumes that eye movements are replaced by shifts of an internal attention mechanism which processes successive features of the pattern. Noton (1970) has proposed an information-processing model of pattern perception expanding on this idea. A fuller theoretical discussion of this model is presented elsewhere (Noton, 1970; Noton \& Stark, 1971a).

Haber and Hershenson (1973, p. 174) state that, with the exception of the work of Gibson and Hochberg and a few others, little evidence is available to guide our thinking about the nature of the construction of an organized visual representation of the world around us. The scanpath is a clear finding of this study, and its occurrence in pattern recognition implicates it as a potential factor in the recognition process. However, this study was the first attempt to systematically examine the occurrence and use of scanpaths during recognition of patterns. Much additional research is needed to elaborate the theoretical role of scanpaths in the processing of visual information.

\section{REFERENCES}

Egeth, H. E. Parallel versus serial processes in multidmensional stimulus discrimination. Perception \& Psychopbysics, 1966, 1 . 245-252.

Gould, J. D. Pattern recognition and eye-movement parameters. Perception \& Psychophysics, 1967, 2, 399-407.

Gould, J. D.. \& Dill, A. B. Eye-movement parameters and pattern discrimination. Perception \& Psychophysics, 1969,6, 311-320.

Haber, R. N.. \& Hershenson, M. The psychology of visual perception. New York: Holt, Rinehart \& Winston, 1873.

Hebb, D. O. The organization of behavior. New York: Wiley, 1049.

Hochberg, J. In the mind's eye. In R. N. Haber (Ed.), Contemporary theory and research in visual perception. New York: Holt, Rinehart \& Winston, 1968.

Kirk, R. E. Experimental design: Procedures for the behavioral sciences. Belmont, Calif: Brooks/Cole, 1968.

Kundel, H. L., \& Nodine, C. F. A computer system for processing eye movement records. Behavior Research Methods \& Instrumentation, $1973,5,147-152$.

Nodine, C. F., \& Steuerle, N. J. Development of perceptual and cognitive strategies for differentiating graphemes. Journal of Experimental Psy chology, 1073,97, 158-166.

Noton, D. A. A theory of visual pattern perception. IEEE Transactions on Systems Science \& Cybernetics, 1970, 6, 340-357.

Noton, D., \& Stark, L. Scanpaths in saccadic eye movements while viewing and recognizing patterns. Vision Research, $1071 a, 2,920-942$.

Noton, D., \& Stark, L. Eye movements and visual perception. Scientific American, 1971b. 224, 35-43.

Vitz, P. C., \& Todd, T. C. A model of the perception of simple geometric figures. Psychological Review, 1971, 78, 207-228.

Winer, B. J. Statistical principles in experimental design. New York: McGraw-Hill, 1962.

Yarbus, A. L. Eye movements and vision. New York: Plenum Press, 1067.

\section{NOTE}

1. An examination of the initial fixations of all scanning patterns showed the following distribution of initial fixations in each of the quadrants of the stimulus display : $26 \%$ in the upper left quadrant: $30 \%$ in the upper right quadrant: $23 \%$ in the lower left quadrant; and $21 \%$ in the lower right quadrant.

(Received for publication May 14, 1973; revision received November 14,1973 .) 